\title{
BI-Scale Fractional Order Statistics for Blind Channel Estimation in Chaotic STBC Systems
}

\author{
Girija Sakimalla Prabhakar ${ }^{1 *}$ \\ ${ }^{1}$ Kakatiya Institute of Technology and Science, Warangal, Telangana, India \\ *Corresponding author's Email: spgirija0515@gmail.com
}

\begin{abstract}
Space-Time Block Coding (STBC) is a MIMO based approach which achieves high reliability and diversity by transmitting multiple copies of data stream across antennas. In this paper, bi-scale based fractional order statistics for blind channel estimation in chaotic STBC systems are proposed. Accordingly, the proposed communication system contains chaotic modulator and transmitter in the transmitter section. Then, the receiver contains the channel equalizer and chaotic demodulator. In the channel equalizer, the signal is transmitted through a channel which is estimated through the proposed channel estimation algorithm. In the proposed channel estimation, the weight updating formulae will be modified based on the comparison of the estimated weighted values and its old weighted value. The analysis of the technique is carried out by finding out the evaluation metrics of BER, MSE and SER with respect to SNR and different antenna array sizeof 4, 5 and 6.
\end{abstract}

Keywords: Chaotic Systems; Space-Time Block Coding; Bi-scale Fractional Order Statistics; Channel Estimation.

\section{Introduction}

It is really cheering that wireless systems have been made use of abundantly throughout the length and breadth of the cosmos to cater to the everrocking needs of the inquisitive human being and the sophisticated machines to exchange communications in between them, regardless of the places in which they are situated [1,2]. Wireless systems generally are home to a transmitter, a radio channel and a receiver and are characterized by the number of their inputs and outputs [3, 4]. As far as a traditional digital communication system is concerned, data finds itself carried away from one place to the other by mapping bit sequences to symbols, and symbols to sample functions of analogue waveforms, which invariably represent weighted sums of one or more sinusoids. Nevertheless, during the period when a sinusoidal carrier is deployed, the diffused power is focused in a narrow band, which inevitably paves the way for the incidence of grave deficiencies as detailed below: 1. Elevated shrinking over narrow frequency bands; 2. elevated levels of intervention with other clients; 3. the accelerated possibility of interception of narrow band signals ; 4. the illegal reception is not at all complicated as only a restricted a-priori awareness is needed for demodulation $[5,6]$.

It is pertinent to note that the Maximum Likelihood based receivers are able to achieve the superlative system performance with the highest diversity and least bit error ratio (BER) though it requires the highly intricate detection mechanism. In this regard, Zero Forcing (ZF) receivers and Minimum Mean Square Error (MMSE) receivers belong to the family of linear receivers [7, 8]. Literature offers two significant universal methods for channel evaluation in MIMO systems, such as the Blind and non-blind methods [9]. Space-Time Codes (STCs) have been extensively and fruitfully initiated in cellular communications together with wireless local area networks. A host of coding schemes have been kick-started in the sector which include orthogonal STBC [10], quasi-orthogonal STBC) [11] and non-orthogonal STBCs. With the intention of keeping at bay the demerits of MLbased channel evaluation techniques, a flood of innovative methods including Second-Order 
Statistics (SOS) [12, 13] and higher order statistics were flagged off. However, it is unfortunate that these novel techniques have found their waterloo in extracting the channel in a full-blind context. A ray of hope to overshoot the constraints of SOS and subspace techniques has made its august appearance in the garb of Fractional-Order Statistics (FOS).

Especially, in chaotic communication systems, symbols are generally mapped into sample functions but disorganized attractors, and the chaotic signals generated by them are communicated as the data carrier [5].The term chaotic communication broadly represents a counterintuitive event; the hypothesis of chaos in communication was kick-started to perk up the communication safety devoid of the escalation in the system intricacy [14]. These chaotic communication schemes possess certain vantage points in relation to the traditional narrow band communication systems [15]. As far as orthogonally facet is concerned, signals produced by several generators or by the same generator with diverse primary situations may be deemed to be uncorrelated (orthogonal), which can be the foundation of the signal separations $[6,16]$.

In this paper, bi-scale fractional order based channel estimation is carried out using chaotic signals. The proposed system contains chaotic modulator and transmitter in the transmitter section. Subsequently, the signal is transmitted through a channel which is estimated by use of bi-scale fractional order statistics. The weight updating formulae is modified based on the comparison of the estimated weighted values and its old weighted value.

The rest of the paper is organized as follows: A brief review of researches related to the proposed technique is presented in section 2. Existing problems are given in section 3 and proposed channel estimation technique is given in section 4. Results and discussions are section 5 and finally, conclusion is summed up in section 6 .

\section{Related Works}

A host of efforts have been made in the literary arena in respect of channel evaluation in STBC systems, a few of which are reviewed here. M. HuanFei and K. HaiBin [5] came out with a bigbang digital communication technique, which predominantly employed chaotic signals in accordance with the adaptive synchronization and parameter identification approach of chaotic systems reported of late. In view of the multi signal nature, the technique deftly blended the chaotic signal communication with the MIMO channel approach in a wireless atmosphere, which followed a totally divergent path vis-a-vis the conventional chaotic communication structures.

S. Mukherjee et al. [6] were highly instrumental in assessing various chaotic digital modulation schemes. They estimated the chaotic carrier employed in some sort of modulation-demodulation techniques twinkling with distinctive qualities which empowered them for employment in safe and multiuser communications. They were also successful in finding the application of Fourier assessment hypothesis in chaos and its traits. L. Moraleset al. [16] elegantly green-signalled two signal recognition techniques taking cues from the Differential Chaos-Shift-Keying (DCSK) modulation which is amazingly endowed with the robust quality of recognizing individual users in jam-packed networks. The technique came out in flying colours in negating interference from intercell clients in the course of the training session and was able to recover the channel state data effectively.

Y. Fang et al. [17] proficiently brought to limelight a MIMO relay differential chaos shift keying cooperative diversity (DCSK-CD) system as an all-inclusive cooperation method, in which both the relay and destination effectively utilized manifold antennas to reinforce the heftiness against signal fading in a wireless network. J. K. Bhalania et al. [4] invested sweat and blood in bringing out two combined semi-blind channel evaluation and data recognition methods and carried out incessant research for Alamouti coded single-carrier (SC) multiple-input multiple-output (MIMO) communication system using Rayleigh flat fading channel model. M. K. Hedayati et al. [18] got wide acclaim for spearheading the semi-blind channel evaluation and symbol recognition in accordance with the space-alternating generalized expectationmaximization (SAGE) technique. It was intended for space time block coded (STBC) multiple input multiple output orthogonal frequency division multiplexing (MIMO-OFDM) systems. At the receiver, the communicated signals of all the clientele were supplemented. By deft deployment of the SAGE approach, superimposed received signals were decayed into their signal segments. Thereafter, the channel was subjected to evaluation at pilot positions.

\section{Existing Drawbacks and Inspiration}

It is only natural that the glaring defects in the training based approaches and differential techniques resulted in the ultimate development to the blind channel evaluation for STBC systems. 
Maximum-likelihood based approaches were the ones employed in the initial stages, but they were found plagued by elevated computational expenses, which paved the way for the origin and growth of Second Order Statistics which also needed adaptation to transmitter. When used, the Higher Order Statistics (HOS) were also found languishing from the deficiencies of elevated BER and SER. The ray of hope originates from the fact that the efficiency in execution of STBC mechanism in MIMO systems can be rocked up by means of employing the chaotic signal generator/modulator in the transmitter section. This is on account of the undeniable fact that chaotic signals are endowed with superlative correlation qualities which are instrumental in setting the stage for the significant surge in outcomes.

There is a plethora of plus points which chaotic communication enjoys when compared to the conservative narrow band communication. The reason is that chaotic signals are well-equipped with an incessant spectrum capable of being employed to overshoot channel deficiencies, especially the narrow-band impacts in the form of frequencyselective fading or narrowband turbulences. Moreover, there is another feather in their cap that the chaotic signals are innately non-periodic and uneven with an intricate configuration which puts several insurmountable roadblocks in the pathway of one who proceeds to assess the configuration of the generator and also to forecast the signals over longer time-frame. The signals produced by several generators or by the same generator with diverse primary conditions may be deemed to be uncorrelated (orthogonal), which can be the foundation of the signal separations.

\section{Proposed Bi-Scale Fractional Order Statistics for Blind Channel Estimation in Chaotic STBC Systems}

Space-Time Block Coding (STBC) is a MIMO based technique which obtains high reliability and diversity by transmitting multiple copies of data stream across antennas. In this paper, bi-scale fractional order based channel estimation is carried out. Here, the performance of STBC system in MIMOis improved by the use of chaotic signals. The proposed system contains chaotic modulator and transmitter in the transmitter section. Subsequently, the signal is transmitted through a channel which is estimated by use of bi-scale fractional order statistics. The weight updating formulae is modified based on the comparison of the estimated weighted values and its old weighted value. Finally, the receiver having the channel equalizer and chaotic demodulator receives the signal.

\subsection{Chaotic STBC System Model}

In this paper, chaotic STBC system is considered. In a conventional digital communication system, data is transmitted from one location to another by mapping bit sequences to symbols, and symbols to sample functions of analog waveforms, which are weighted sums of one or more sinusoids. Generally, in chaotic communication systems, symbols are no longer mapped into sample functions but chaotic attractors, and the chaotic signals produced by chaotic attractors are transmitted as the information carrier. Such chaotic communication schemes have several advantages over conventional narrow band communication systems.

We have assumed that the system transmits $k$ symbols through $k_{t r}$ transmitter antennae and is received through $k_{r e}$ antennas. Let the channel matrix be represented by $R$. The system model [19] is given in figure 1 .

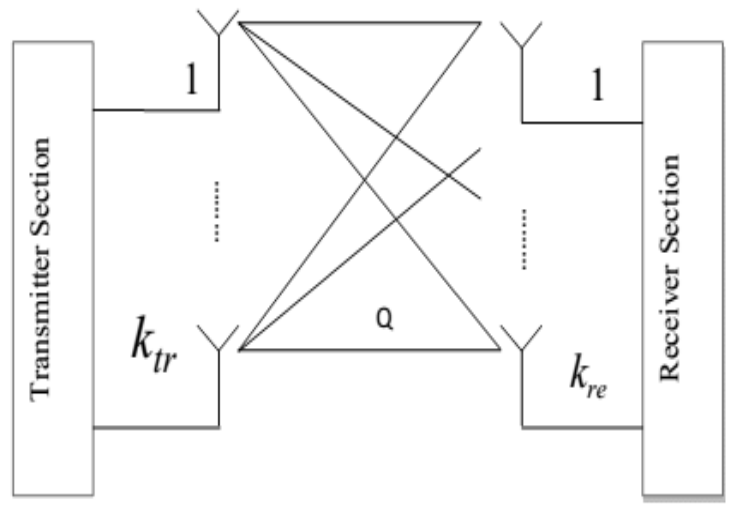

Figure.1 The system model

In this paper, the matrices are represented by upper case letters and lower case for scalars. Transpose of a matrix $\mathrm{X}$ is represented by $X^{T}$ and Hermitian of the matrix $\mathrm{X}$ is represented by $X^{H}$. Complex conjugate of a matrix $\mathrm{X}$ is represented by $X^{*}$ and real and imaginary parts are represented by $\operatorname{Re}(X)$ and $\operatorname{I} m(X)$ respectively. Kronecker product is represented by the operator $\otimes$ and expectation operator is given by $\mathrm{E}$. The identity and zero matrices of order $\mathrm{k}$ is represented by $1_{k}$ and $0_{k}$. The unit vector $y n_{j}{ }^{(k)}$ is an $\mathrm{k}$-dimensional row vector having 1 only at $j^{\text {th }}$ place and 0 elsewhere. 
Elementary matrix $Y N_{p q}{ }^{(k)}$ is matrix having dimension of $k \times k$, of which $p q$ element is 1 and 0 elsewhere. $p q$ element is the element at $p^{\text {th }}$ row and $q^{\text {th }}$ column.

There are many Choas based coherent and noncoherent modulation schemes. Out of which Antipodal Chaos Shift Keying (ACSK) [6] performs better because of their noise robust performances. It offers best noise performance in both AWGN and fading environment and requires chaos synchronization at receiving end.

The system is considered which transmits $k$ symbols in $t$ time slots. The space time block encoder generates block matrix (of dimension $k_{t r} \times t$ ) from of symbols represented by $x=\left[x_{1}, x_{2}, \ldots, x_{k}\right]^{T}$. ACSK is described by a single basis function $x(t)$. The waveform corresponding to binary bit is given as:

$$
C(t)=\left\{\begin{array}{l}
\sqrt{E n} x(t) \text { for bit ' } 1 \text { ' } \\
-\sqrt{E n} x(t) \text { for bit ' } 0 \text { ' }
\end{array}\right.
$$

Here, En represents the bit energy. The modulator and the demodulator of the ACSK [6] can be given by the figures 2 and 3 .

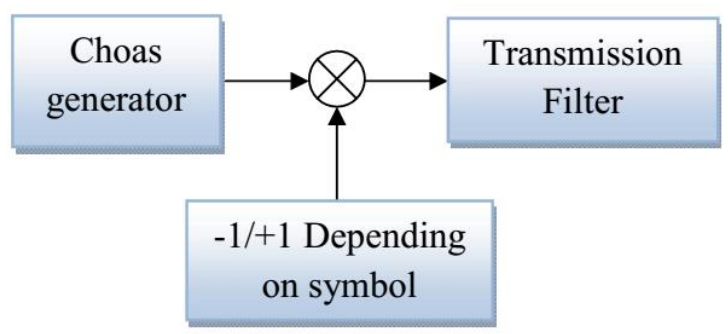

Figure.2 Block diagram of the ACSK modulator

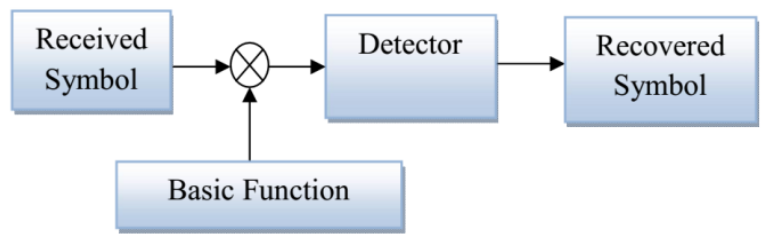

Figure.3 Block diagram of the ACSK demodulator

The signals are received in the receiver section, which is composed of $k_{r e}$ antenna elements in the array. The channel in between the receiver and the transmitter is modelled as a quasi-static frequencyflat channel. In the receiver, thecorrelator output can be given by:

$$
Q_{k}(t)=\int_{0}^{t}\left[\tilde{c}_{k}(t)+n(t)\right] x(t) d t
$$

Where, $n(t)$ is the noise in channel and $\tilde{c}_{k}(t)$ is the received version of the transmitted signal. The correlator output is sampled before it is applied to the threshold detector. The first term in the correlator output determines overall correlation level, and zero threshold level is applied for detecting bits.

\subsection{Channel Estimation Section}

This section covers the channel estimation which comprises of data whitening, computation of unitary matrix and calculation of the expression of the gradient in the Euclidean space. It also details of Kurtosis- based cost function.

At first, Second Order Statistics (SOS) [19] is applied for the channel estimation for a unitary matrix. The transmitted symbols (represented as $x$ ) is assumed to be non-Gaussian, independent and identically distributed. Hence, the average transmits power on each antenna is normalized to onewhich can be expressed as: $E\left[C\left(x_{i}\right) C^{H}\left(x_{i}\right)\right]=t 1_{k_{t r}}$.

Hence, the covariance matrix of the noiseless transmitted signals $\left(S=E\left[Q_{i} Q_{i}{ }^{H}\right]-t \sigma^{2} 1_{k_{r e}}\right)$ can be simplified as;

$$
S=R E\left[C\left(x_{i}\right) C^{H}\left(x_{i}\right)\right] R^{H}=t R R^{H}
$$

The matrix can be decomposed to form $S=Z \lambda Z^{H}$ using singular decomposition. Here, $Z$ having dimension $k_{r e} \times k_{t r}$ is such that $Z^{H} Z=1_{k_{t r}}$ and $\lambda$ is a diagonal matrix having dimension of $k_{t r} \times k_{t r}$. After the decomposition, we can write as:

$$
R=\frac{1}{\sqrt{t}} Z \lambda^{\frac{1}{2}} Y^{H}
$$

The whitened data block can be written as:

$$
B_{i}=\sqrt{t} Z \lambda^{-\frac{1}{2}} Y^{H} Q_{i}
$$

Consequently, whitened data block can be represented as linear zero-forcing decoder. Hence, we have:

$$
B_{i}=Y^{H} C\left(x_{i}\right)+\sqrt{t} Z \lambda^{-\frac{1}{2}} Y^{H} N_{i}
$$

The second term of the above expression can be observed as a multi-dimensional zero-mean Gaussian signal which can be denoted by $V_{i}$ having dimension of $k_{t r} \times t$. The real valued column vector $\tilde{x}_{i}$ I can be defined as: 


$$
\tilde{x}_{i} \Delta=\left[\begin{array}{c}
\operatorname{Re}\left[x_{1}^{(i)}\right] \\
\ldots \\
\operatorname{Re}\left[x_{k}{ }^{(i)}\right] \\
\operatorname{Im}\left[x_{1}{ }^{(i)}\right] \\
\ldots \\
\operatorname{Im}\left[x_{k}{ }^{(i)}\right]
\end{array}\right]
$$

Using the vector operator of $\vartheta\{\}$ and by using equations (1) and (6):

$$
\tilde{b}_{i}=\tilde{Y} \Lambda \tilde{x}_{i}+\tilde{v}_{i}
$$

Here, $\tilde{b}_{i}$ and $\tilde{v}_{i}$ are $2 k_{t r} t$ dimensional column vectors and $\Lambda$ has the dimension of $2 k_{t r} t \times 2 k$. These matrices are defined as:

$$
\begin{aligned}
\tilde{b}_{i} & \underline{\Delta}\left[\begin{array}{l}
\operatorname{Re}\left(\vartheta\left\{B_{i}{ }^{H}\right\}\right. \\
\operatorname{Im}\left(\vartheta\left\{B_{i}{ }^{H}\right\}\right.
\end{array}\right] \\
\tilde{v}_{i} & \triangleq\left[\begin{array}{c}
\operatorname{Re}\left(\vartheta\left\{V_{i}^{H}\right\}\right. \\
\operatorname{Im}\left(\vartheta\left\{V_{i}^{H}\right\}\right.
\end{array}\right] \\
\Lambda & \triangleq \\
& =\left[\begin{array}{cc}
\operatorname{Re}\left(\vartheta\left\{\gamma_{1}{ }^{H}\right\}\right) \ldots \operatorname{Re}\left(\vartheta\left\{\gamma_{2 k}{ }^{H}\right\}\right) \\
\left.\operatorname{Im}\left(\vartheta \gamma_{1}{ }^{H}\right\}\right) \ldots \operatorname{Im}\left(\vartheta\left\{\gamma_{2 k}{ }^{H}\right\}\right)
\end{array}\right] \\
\tilde{Y} & =\left[\begin{array}{cc}
\operatorname{Re}\left(Y^{T}\right) \otimes 1_{t} & -\operatorname{Im}\left(Y^{T}\right) \otimes 1_{t} \\
\operatorname{Im}\left(Y^{T}\right) \otimes 1_{t} & \operatorname{Re}\left(Y^{T}\right) \otimes 1_{t}
\end{array}\right]
\end{aligned}
$$

The ZF equalizer [19] computed the inverse matrix to compensate channel and space-time coding to have:

$$
\left[\begin{array}{c}
\operatorname{Re}\left[x_{1}{ }^{(i)}\right] \\
\ldots \\
\operatorname{Re}\left[x_{k}{ }^{(i)}\right] \\
\operatorname{Im}\left[x_{1}{ }^{(i)}\right] \\
\ldots \\
\operatorname{Im}\left[x_{k}{ }^{(i)}\right]
\end{array}\right]=\Lambda^{\prime} \tilde{y}^{T} \tilde{b}_{i}
$$

Here, $\Lambda^{\prime}$ represents the pseudo-inverse of matrix $\Lambda$. Let $\tilde{x}_{j}^{(i)}$ represents the $i^{t h}$ estimated symbol of the $j^{\text {th }}$ block represented by:

$$
\tilde{x}_{i}^{(j)}=\left[y n_{j}^{(i)} y n_{j}^{(i)}\right] \Lambda^{\prime} \tilde{Y}^{T} \tilde{b}_{i}
$$

The estimation of the unitary matrix is carried out by maximizing the statistical independence of the Zero-Forcing equalized symbols $\left(x_{i}^{(j)}\right)$. Kurtosis represented $\Gamma$ is a measure of non-guassianity which is employed to maximize the statistical independence of $x_{i}^{(j)}$. Un-normalized Kurtosis given by:

$$
\Gamma(x) \stackrel{\Delta=}{=}\left[|x|^{4}\right]-2\left(E\left[|x|^{2}\right]\right)^{2}-E[x x] E\left[x^{*} x^{*}\right]
$$

The estimate of unitary matrix $Y$ can be computed by maximizing the function of
$\sum_{j=1}^{k}\left|\Gamma\left(\tilde{x}_{j}^{(i)}\right)\right| . G(Y)$ is a real-valued cost function that is dependent on the matrix $Y$. The estimate of $Y$ represented by $\tilde{Y}$ can be expressed as:

$$
\tilde{Y}:\left\{\begin{array}{c}
\min _{y} G(Y)=\sum_{j=1}^{k}\left|\Gamma\left(\tilde{x}_{j}^{(i)}\right)\right| \\
\text { subjectto } Y Y^{H}=1_{k_{t r}}
\end{array}\right.
$$

\subsection{Bi-Scale Fractional Order based Steepest Descent Model}

The minimization of the cost function is performed using steepest descent method [20]. Here, initially the gradient matrix is defined by:

$$
\Delta \delta=\frac{d G(Y)}{d Y^{*}}
$$

Where, $G(Y)$ is the cost function and $\Delta \delta$ is the gradient of cost function. Now, $\frac{d G(Y)}{d Y^{*}}$ can be approximated as:

$$
\frac{d G(Y)}{d Y^{*}} \triangleq \frac{1}{=}\left(\frac{d G(Y)}{d \operatorname{Re}(Y)}+\frac{d G(Y)}{d \operatorname{Im}(Y)}\right)
$$

Where, $\operatorname{Re}(Y)$ and $\operatorname{Im}(Y)$ are the real and imaginary parts. Let the element at the $m^{\text {th }}$ row and $n^{\text {th }}$ column of the $Y$ matrix be denoted by $y_{m n}$. By comparing the above two equations, we have:

$$
\Delta \delta=\sum_{m=1}^{k_{t r}} \sum_{m=1}^{k_{t r}} \frac{1}{2} Y N_{m n}^{k_{t r}}\left(\frac{d G(Y)}{d \operatorname{Re}\left(y_{m n}\right)}+\frac{d G(Y)}{d \operatorname{Im}\left(y_{m n}\right)}\right)
$$

As $\min _{y} G(Y)=\sum_{i=1}^{k} \Gamma\left|\left(\tilde{x}_{i}^{(j)}\right)\right|$, we can write equation as:

$$
\Delta \delta=\sum_{m=1}^{k_{t r}} \sum_{n=1}^{k_{t r}} \sum_{i=1}^{k} \frac{1}{2} Y N_{m n}^{k_{t r}}\left(\frac{d \Gamma\left|\left(\tilde{x}_{i}^{(j)}\right)\right|}{d \operatorname{Re}\left(y_{m n}\right)}+\frac{d \Gamma\left|\left(\tilde{x}_{i}^{(j)}\right)\right|}{d \operatorname{Im}\left(y_{m n}\right)}\right)
$$

Here, $\tilde{x}_{i}^{(j)}$ represents the $i^{\text {th }}$ estimated symbol of the $j^{\text {th }}$ block and $\Gamma$ gives the Kurtosis function.

Once the gradient matrix is computed, the steepest descent algorithm based on constrain minimization is employed. In each iteration, $Y$ value is modified in the course of the negative gradient. Afterwards, a symmetric orthogonalization is employed to reinstate the unitary constraint of $Y$.

The description of the process [19] is given below. At first, covariance matrix of the noiseless 
transmitted signal $S$ is computed using equation (3). Then, Eigen value decomposition $\left(S=Z \lambda Z^{H}\right)$ is carried out and whitened data $B_{i}$ is computed using equation (5). Arbitrarily initialize the unitary matrix $Y$.

Once above mentioned procedures are carried out, execute the following iteration until the termination condition is satisfied. Initially assign $G(Y)$ to $G_{\text {old }}$. Compute the gradient $\Delta \delta$ in the Euclidean space. Modify the value of $Y$ as $Y-\mu \Delta \delta$ so that $Y_{t+1}=Y_{t}-\mu \Delta \delta$ where $\mu$ is the step size. Update the value of $Y$ as $Y\left(Y^{H} Y\right)^{-1 / 2}$.

The termination criteria condition is given by $G_{\text {old }}-G(Y)<$ Threshold. Finally after the iterations, the channel matrix $R$ is calculated (equation 4).This results channel estimation for STBC systems. Employment of fractional order statistics can overcome from major disadvantages like that it can lead to undesired suboptimal solutions. Bi-scale based fractional order can furthermore improve systems and is applied in the weight updating step. We already know:

$$
Y_{t+1}=Y_{t}-\mu \Delta \delta
$$

The equation can be re-written as:

$$
Y_{t+1}-Y_{t}=-\mu \Delta \delta
$$

As the left side is the discrete version of the derivative of order $\alpha=1$, it can be written as:

$$
B^{\alpha}\left[Y_{t+1}\right]=-\mu \Delta \delta
$$

Applying fractional order fractional calculus:

$$
\begin{aligned}
& B^{\alpha}\left[Y_{t+1}\right]=Y_{t+1}-\alpha Y_{t}-\frac{1}{2} \alpha Y_{t-1}-\frac{1}{6} \alpha(1-\alpha) Y_{t-2}- \\
& \frac{1}{24} \alpha(1-\alpha)(2-\alpha) Y_{t-3}
\end{aligned}
$$

Substituting equation (24) in equation (23):

$$
\begin{aligned}
& Y_{t+1}-\alpha Y_{t}-\frac{1}{2} \alpha Y_{t-1}-\frac{1}{6} \alpha(1-\alpha) Y_{t-2}- \\
& \frac{1}{24} \alpha(1-\alpha)(2-\alpha) Y_{t-3}=-\mu \Delta \delta
\end{aligned}
$$

By re-arranging, we have:

$$
\begin{aligned}
& Y_{t+1}=\alpha Y_{t}+\frac{1}{2} \alpha Y_{t-1}+\frac{1}{6} \alpha(1-\alpha) Y_{t-2}+ \\
& \frac{1}{24} \alpha(1-\alpha)(2-\alpha) Y_{t-3}-\mu \Delta \delta
\end{aligned}
$$

By the use of bi-scale based fractional order results in having two solutions based on the cost function obtained. Based on the obtained cost functions, the step size is varied and the step sizes in consideration are $\mu_{1}$ and $\mu_{2}$. Suppose the cost functions obtained from $G_{1}$ and $G_{2}$, we can re-write the equation based on the bi-scale fractional order as:

$$
\begin{aligned}
& \text { if } G_{1} \geq G_{2}: Y_{t+1}=\alpha Y_{t}+\frac{1}{2} \alpha Y_{t-1}+\frac{1}{6} \alpha(1-\alpha) Y_{t-2}+ \\
& \frac{1}{24} \alpha(1-\alpha)(2-\alpha) Y_{t-3}-\mu_{1} \Delta \delta \\
& \quad \text { if } G_{1}<G_{2}: Y_{t+1}=\alpha Y_{t}+\frac{1}{2} \alpha Y_{t-1}+\frac{1}{6} \alpha(1-\alpha) Y_{t-2} \\
& \quad+\frac{1}{24} \alpha(1-\alpha)(2-\alpha) Y_{t-3}-\mu_{2} \Delta \delta
\end{aligned}
$$

Equations 27 and 28 would be used instead of the normal weight updating equation in the steepest descent algorithm which was discussed earlier. Then, the receiver contains the channel equalizer and chaotic demodulator. In the channel equalizer, the signal is transmitted through the channel which is estimated through the proposed channel estimation algorithm.

The pseudo code of the proposed technique can be given as follows:

\section{Results and Analysis}

The proposed technique results are discussed and analysed in this section. Section 5.1 describes the implementation and evaluation metric details. Section 5.2 gives the performance analysis.

\subsection{Implementation Details and Evaluation Metrics}

For implementing the proposed technique, we have used Mat lab version (7.12). This proposed technique is done in windows machine having Intel i-7 processor with speed $3.2 \mathrm{GHz}$ and $6 \mathrm{~GB}$ RAM. The evaluation metrics employed are SER (Symbol Error Rate) [21], BER (Bit Error Rate) [22] and MSE (Minimum Squared error) [22].

Bit Error Rate (BER) is defined as the rate at which errors occur in a transmission system. BER is the ratio of error-bits received to the total bits sent. Symbol Error Rate (SER) is defined as a number erroneously decoder information symbols (constellation points) divided by total number of transmitted symbols. MSE is the difference between values implied by an estimator and the true values of the quantity being estimated. MSE measures the average of the squares of the "errors." The error is the amount by which the value implied by the estimator differs from the quantity to be estimated. The evaluation metric values are taken for varying SNR (Signal to Noise Ratio conditions) [22].

Computation time is also taken as an evaluation metric where time required for the execution of the 
implementation is given. Analysis is also carried out by finding the cost function at various iterations.

\subsection{Performance Analysis}

The performance of the proposed technique is evaluated under various evaluation metrics of BER, MSE and SER with respect to SNR. Comparative analysis is also carried out by comparing to existing prominent technique of $\mathrm{M}$. HuanFei and $\mathrm{K}$. HaiBinscheme [5]. The analysis is carried out for antenna size of 4,5 and 6 .

\section{a) BER curves}

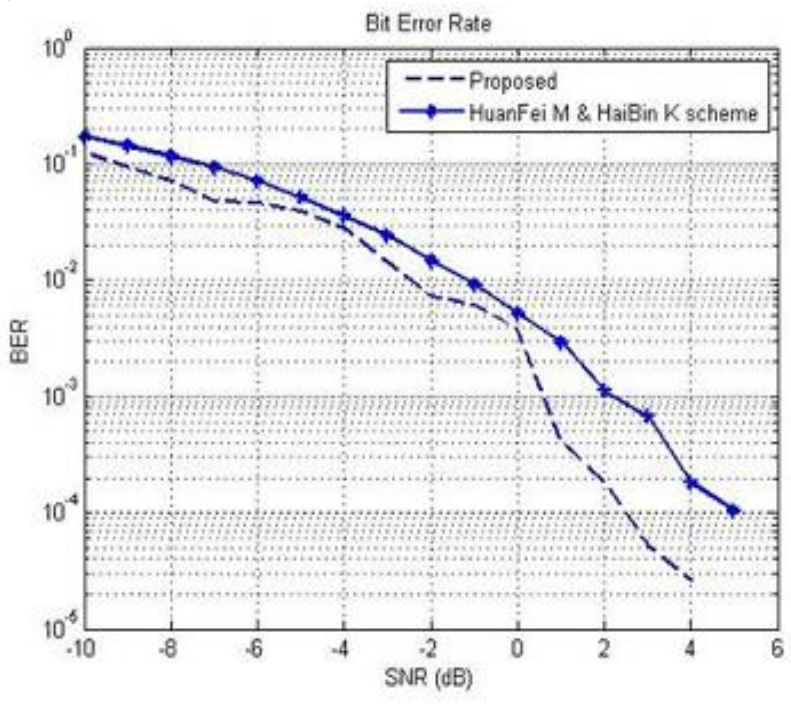

Figure.4 BER vs SNR for antenna size 4

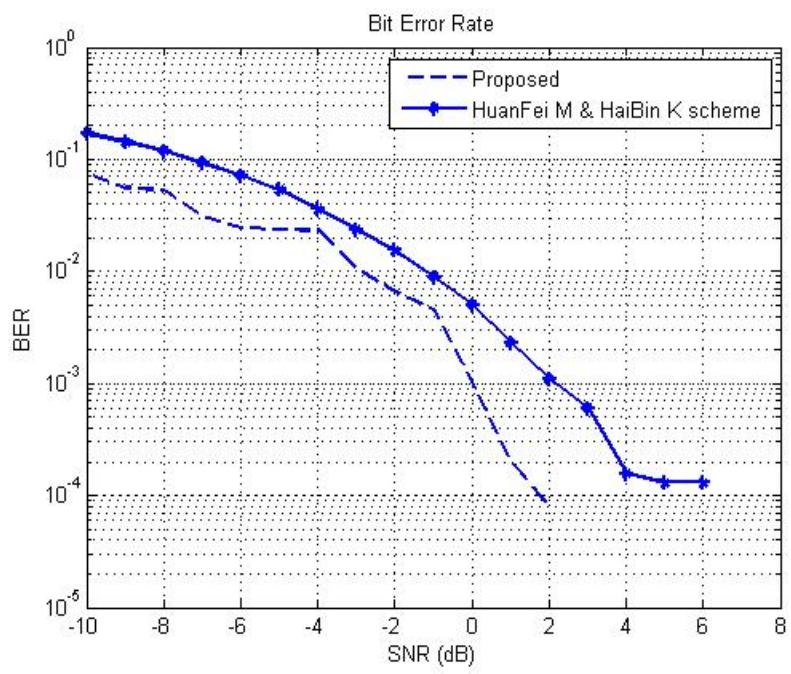

Figure.5 BER vs SNR for antenna size 5

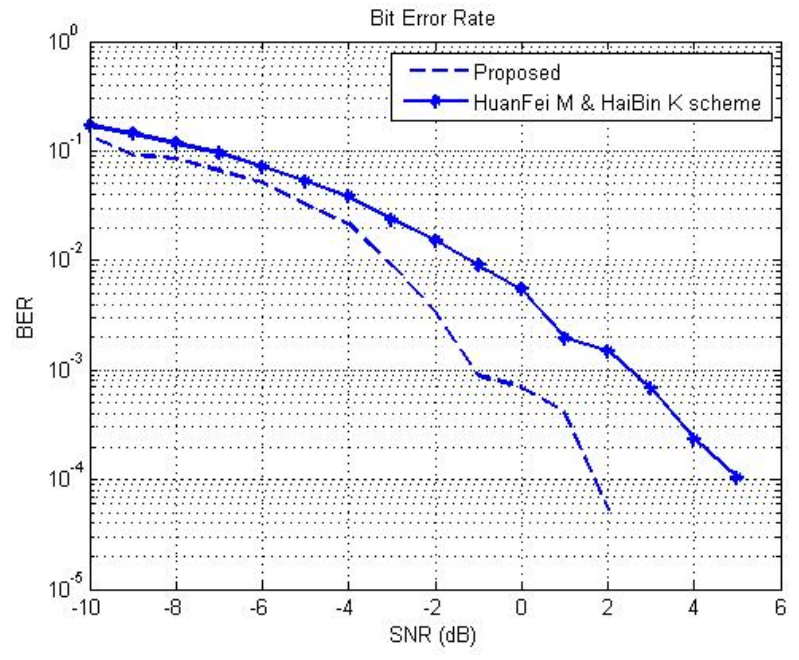

Figure.6 BER vs SNR for antenna size 6

\section{Inferences from figure 4, 5 and 6}

- Figure 3, 4 and 5 gives the BER curve with respect to SNR antenna size of four, five and six respectively.

- Comparative analysis is carried out by comparing to existing prominent technique of M. HuanFei and K. HaiBin [5].

- We can observe that our proposed technique has obtained lower curve indicating effectiveness of the technique.

- Analysing the BER graph for the proposed technique, we can observe BER decreases with increasing SNR.

- We can also observe that the proposed technique achieved better results with increasing the number of antennas.

b) MSE curves

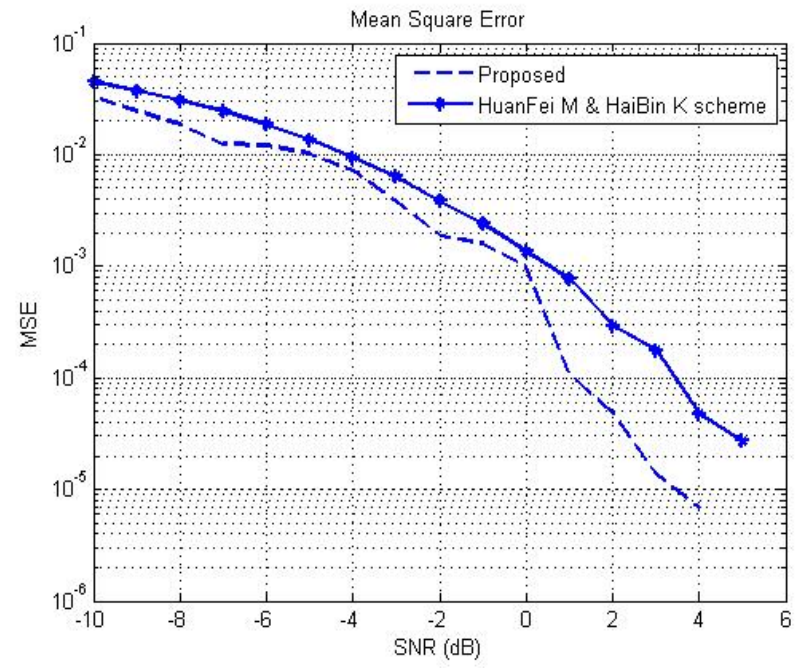

Figure.7 MSE vs SNR for antenna size 4 


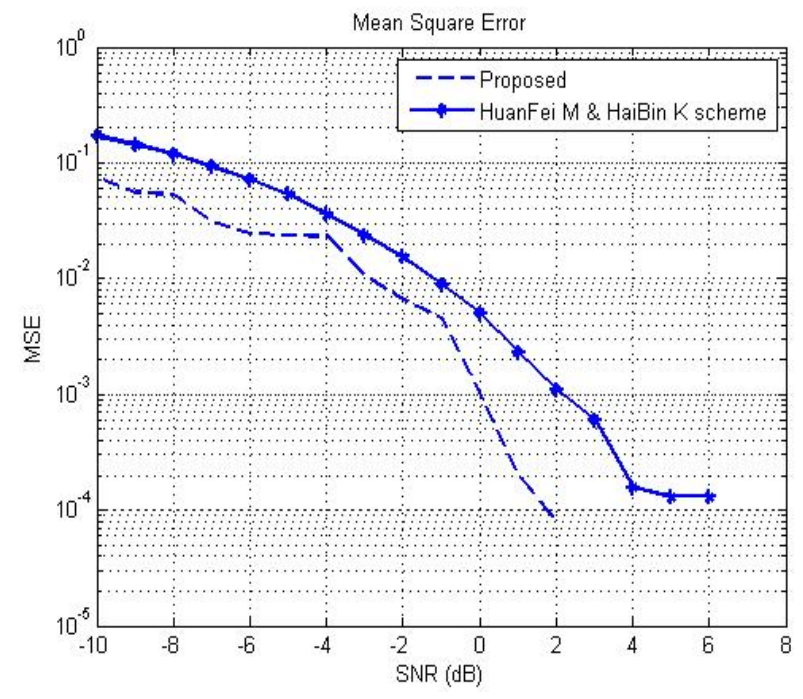

Figure.8 MSE vs SNR for antenna size 5

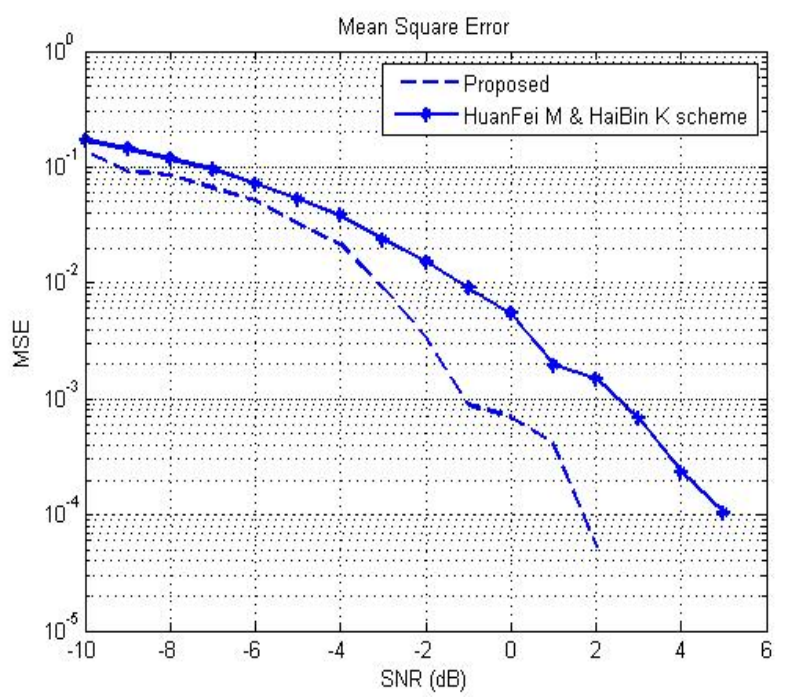

Figure.9 MSE vs SNR for antenna size 6

\section{Inferences from figure 7, 8 and 9}

- Figure 7,8 and 9 gives the MSE curve with respect to SNR antenna size of 4, 5 and 6 respectively.

- In this section, we compare our proposed work with $M$. HuanFei and $K$. HaiBinscheme.

- From the figures, we can observe that our proposed technique has obtained lower curve indicating effectiveness of the technique.

- The MSE graph for the proposed technique decreases with increasing SNR.

c) SER Curve

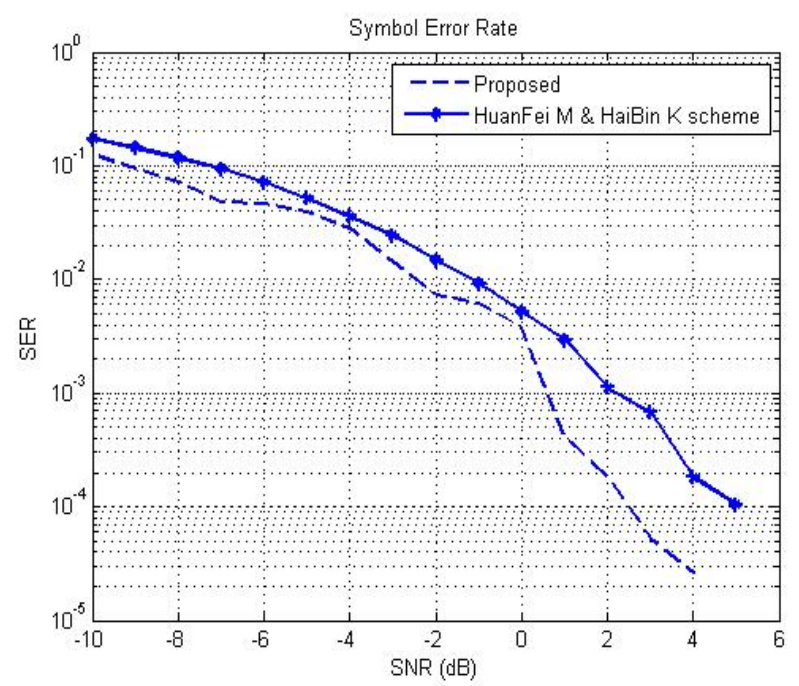

Figure.10 SER vs SNR for antenna size 4

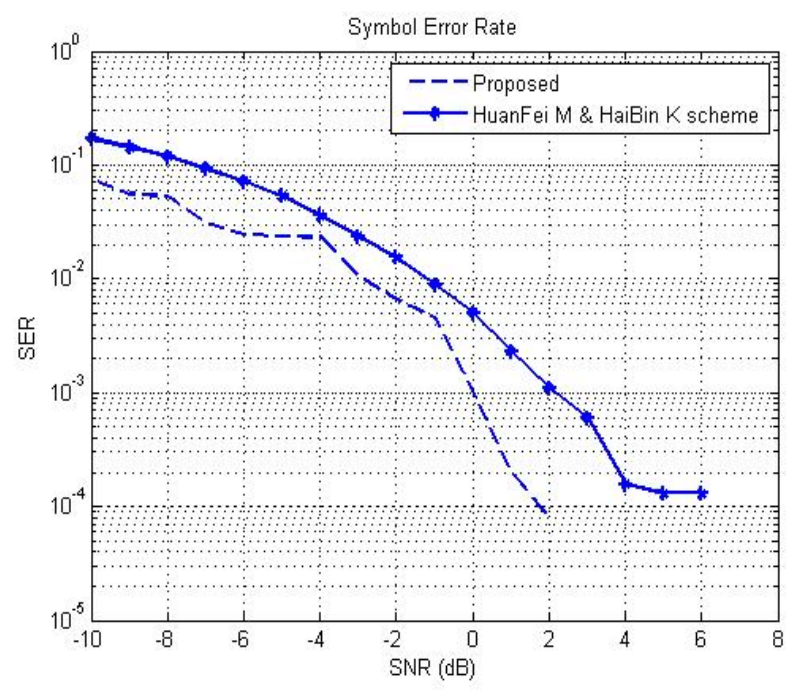

Figure.11 SER vs SNR for antenna size 5

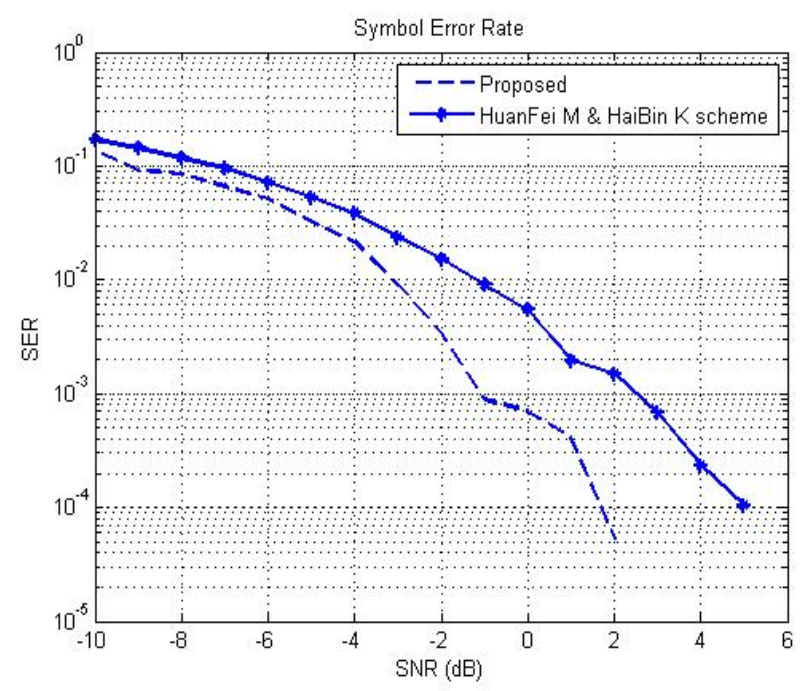

Figure.12 SER vs SNR for antenna size 6 


\section{Inferences from 10, 11 and 12}

- Figure 10, 11 and 12 gives the SER curve with respect to SNR antenna size of 4, 5 and 6 respectively.

- Here, our proposed work compared with the existing work.

- From the figures, we can observe that our proposed technique has obtained lower SER curves showing the effectiveness of the technique.

- We can also observe that SER curve decreases with increasing SNR.

\section{d) Cost function curves}

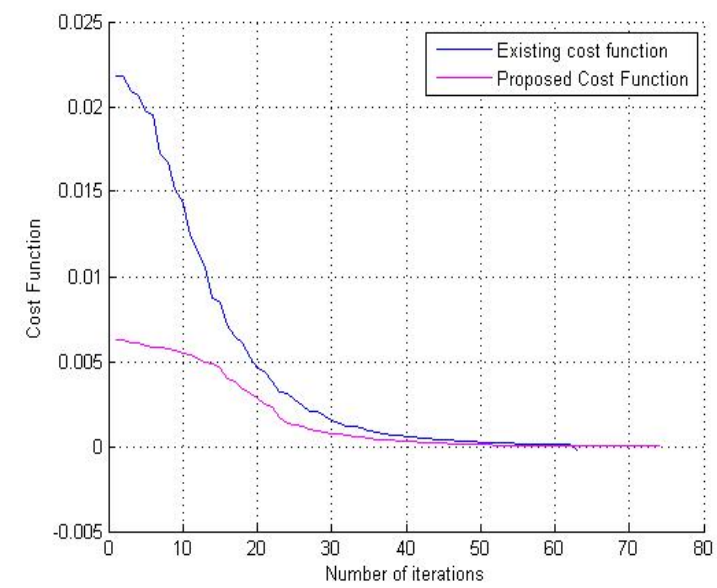

Figure.13 Cost function for antenna size 4

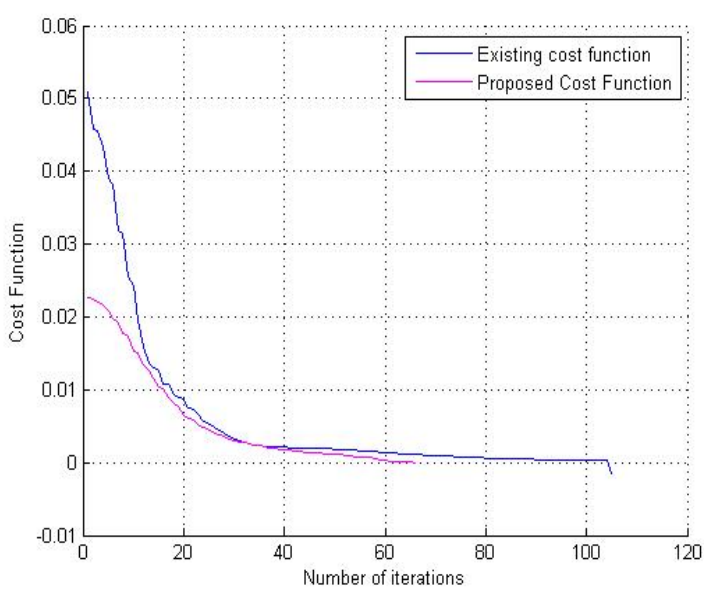

Figure.14 Cost function for antenna size 5

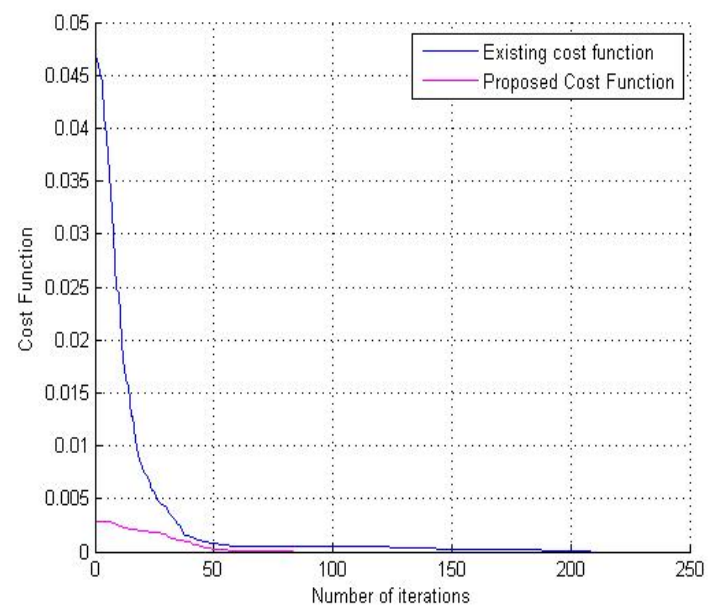

Figure.15 Cost function for antenna size 6

\section{Inferences from 13, 14 and 15}

- Figure 13,14 and 15 gives the cost function curve with respect to number of iterations for antenna size of 4, 5 and 6 respectively.

- Comparative analysis is carried out by proposed cost function to the existing cost function.

- We can also observe that the proposed cost function achieved better values by having higher curve.

- We can also observe that the proposed cost function achieved better results with increasing the number of antennas.

\section{e) Computation Time}

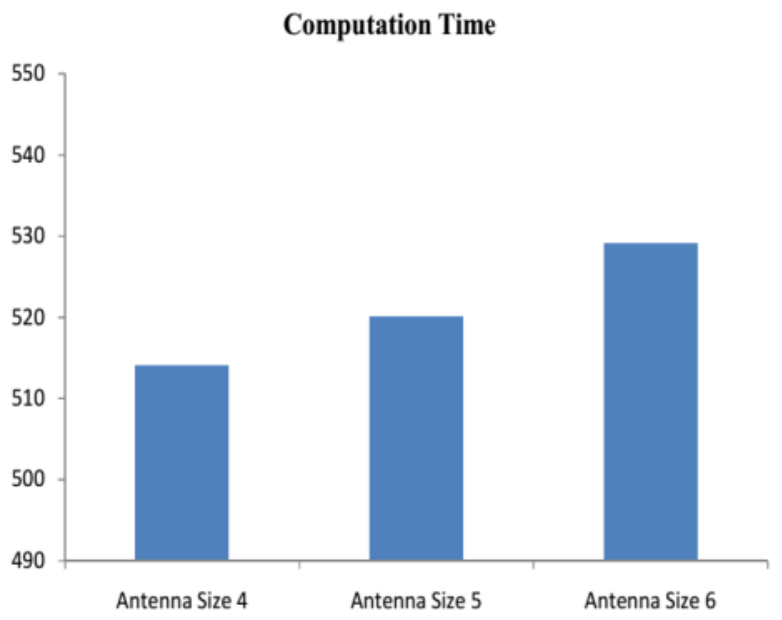

Figure.16 Computation Time

From the above figure 16, we can infer that the proposed technique achieved good response by having low computation time. We can see that with decreasing the antenna size, computation time decreases. The technique achieved an average computation time of $527.8 \mathrm{~ms}$. 


\section{Conclusion}

Bi-scale based fractional order statistics for blind channel estimation in chaotic STBC systems are proposed in this paper. The proposed communication system contains chaotic modulator and transmitter in the transmitter section. Then, the receiver contains the channel equalizer and chaotic demodulator. For channel estimation, bi-scale fractional order statistics-based channel estimation algorithm is proposed. The analysis of the technique is carried out by finding out the evaluation metrics of BER, MSE and SER with respect to SNR and different antenna array size of 4,5 and 6 . The proposed technique achieved good results by obtaining having lower BER, MSE and SER when compared with prominent technique of M. HuanFei and K. HaiBin [5].The technique also achieved low computation time of $527.8 \mathrm{~ms}$.

In future, this work can be carried on further by considering other channel models and evaluating the system performance.

\section{Reference}

[1] G. J. Foschini, M. J. Gans, "On Limits of Wireless Communications in Fading Environments when using Multiple Antennas", Wireless Personal Communications, Vol. 6, No. 3, pp. 311-335, 1998.

[2] R. Kumar and R. Saxena, "Performance Comparison of MIMO-STBC Systems with Adaptive Semi blind Channel Estimation Scheme", Wireless Personal Communications, Vol. 72, No. 4, pp. 2361-2387, 2013.

[3] V. Tarokh, N. Seshadri, and A. R. Calderbank, "Space-Time Codes for High Data Rate Wireless Communication: Performance Criterion and Code Construction", IEEE Trans. Inform. Theory, Vol. 44, No. 2, pp. 744-765, March 1998.

[4] J. K. Bhalania, D. Chauhana, Y. P. Kostab and A. I. Trivedic, "Near Optimal Performance Jjoint Semiblind Channel Estimation and Data Detection Techniques for Alamouti Coded Single-Carrier(SC) MIMO Communication Systems", Physical Communication, Vol. 8, pp. 31-37, September 2013.

[5] M. H. Fei and K. HaiBin, "A New Scheme of Digital Communication using Chaotic Signals in MIMO Channels", Information Sciences, Vol. 55, No. 10, pp. 2183-2193, 2012.

[6] S. Mukherjee, D. Ghosh, D. Nandi and D. Datta, "A Review on Chaotic Digital Modulation Schemes", In proceedings of ICACCT, pp. 66-70, 2013.

[7] Y. Ouyang, C. G. Univ, W. J. Chen and S. H. Wu, "Channel Estimation for MIMO-OFDM Systems using a Novel STBC Pilot Pattern", International High Speed Intelligent Communication Forum, pp. 1$2,2012$.
[8] V. Tarokh, H. Jafarkhani, and A. Calderbank, "Space time Block Codes from Orthogonal Designs", IEEE Trans. Inf. Theory, Vol. 45, No. 5, pp. 744-765, 1999.

[9] T. M. Duman and A. Ghrayeb, "Coding for MIMO Communication System”, John Wiley and sons, 2007.

[10]G. Ganesan and P. Stoica, "Space-time block codes: a maximum SNR approach", IEEE Trans. Inf. Theory, Vol. 47, No. 4, pp. 1650-1656, 2001.

[11]H. Jafarkhani, “A quasi-orthogonal space-time block code", IEEE Trans. Commun., Vol. 49, No. 1, pp. 1-4, 2001.

[12]Larsson, E. G. Stoica, Petre and J. Li, "Orthogonal space-time block codes:maximum likelihood detection for unknown channels and unstructured intereferences", IEEE Trans. Signal Process., Vol. 51, No. 2, pp. 362-372, 2003.

[13]J. Via and I. Santamaria, "Correlation matching approaches for blind OSTBC channel estimation," IEEE Trans. Signal Process., Vol. 56, No. 12, pp. 5950-5961, 2008.

[14]Abel and W. Schwarz, "Chaos CommunicationsPrinciples, Schemes and System Analysis", Proc. IEEE, Vol. 90, No. 5, pp. 691-710, 2002.

[15]A. Abel, W. Schwarz, "Chaos communicationsprinciples, schemes and system analysis", Proc IEEE, Vol. 90, No. 5, pp.691-710, 2002.

[16]L. Morales and S. Roy, "Chaos-based modulation for blind and coherent signal detection in noncooperative TDD cellular networks with large antenna arrays", In proceedings of International Conference on Computing, Networking and Communications (ICNC), pp. 680-685, 2014.

[17] Y. Fang, J. Xu, L. Wang and G. Chen, "Performance of MIMO Relay DCSK-CD Systems over Nakagami Fading Channels", IEEE Transactions on Circuits and Systems I: Regular Papers, Vol. 60 , No. 3, pp. 757-767, 2013.

[18]M. K. Hedayati, H. Bakhshi and M. Cheraghi, "SAGE Algorithm for Semi-Blind Channel Estimation and Symbol Detection for STBC MIMO OFDM Systems", Wireless Personal Communications, Vol. 71, No. 2, pp. 1541-1555, 2013.

[19] V. Choqueuse, A. Mansour, G. Burel, L. Collin and K. Yao, "Blind Channel Estimation for STBC Systems Using Higher-Order Statistics", IEEE Transactions On Wireless Communications, Vol. 10, No. 2, pp.495-505, 2011.

[20]P. Deift, X. Zhou, "A steepest descent method for oscillatory Riemann-Hilbert problem", The Annals of Mathematics, Vol. 137, No. 2, pp. 295-368, 1993.

[21]D. A. Bell, "Information Theory and its Engineering Applications", 3rd edition, New York, Pitman, 1962.

[22]J. Proakis and M. Salehi, "Digital Communications", McGraw-Hill Education, November, 2007.

[23]H. Anzhong, L. Tiejun, G. Hui, Y. Chau and Yang, "Pilot design for large-scale MIMO system with semi-blind channel estimation", IEEE Transactions on Communications, 2013. 